\title{
Réfugiés et sécurité régionale en Afrique : un défi pour le HCR
}

\author{
JACK M. MANGALA
}

\section{Résumé}

Les réfugiés sont devenus intimement liés aux préoccupations de sécurité intérieure et extérieure des États africains. Fruit de l'instrumentalisation politique des réfugiés, ce rapprochement pose un énorme défi en termes de protection et de recherche de solutions. L'étude porte d'abord sur les mécanismes à travers lesquels s'opère ce rapprochement et sur les enjeux que représentent les réfugiés sur le plan de la sécurité. Elle traite ensuite de la réponse du HCR et de la communauté internationale à ce défi et démontre comment l'organisation humanitaire a su trouver, à travers cette délicate question, les voies d'une expansion opérationnelle empreinte d'innovation afin de concrétiser les prescrits $d u$ droit international des réfugiés dans le domaine de la sécurité.

\section{Abstract}

For African states, refugees have become closely linked to internal and external security concerns. This linkage is a result of the political "instrumentalization" of refugees and represents a huge challenge in terms of the protection of refugees and the search for solutions. This study begins by analyzing the mechanics through which this linkage operates and the security stakes that refugees represent. It then proceeds to examine the response of the UNHCR and of the international community to this challenge and shows how - through its handling of this sensitive issue - the humanitarian organization has been able to devise imaginative ways to expand its operations in order to give shape to the rights of refugees in matters of security as prescribed by international refugee law.

\section{Introduction}

$\mathrm{D}$ epuis le début du mois de décembre 2000, la Guinée est l'objet d'attaques armées dans ses frontières sud, qui abritent la plus grande concentration de réfugiés ayant fui les guerres civiles de la Sierra Leone et du Liberia. La question est si préoccupante que le haut-commissaire aux réfugiés nouvellement désigné a consacré à la Guinée son premier voyage africain. La situation guinéenne rappelle, à maints égards, celle qu'a connue l'est de la République démocratique du Congo après le génocide rwandais en 1994. La communauté internationale s'était alors engagée à en tirer les leçons, notamment quant à la sécurité et au mode d'installation des réfugiés. Quatre ans après, l'évolution de la situation en Guinée, qui risque d'embraser toute la sous-région, semble indiquer que cet exercice n'a pas été mené à bien. Si la question de la sécurité a toujours accompagné le débat sur les réfugiés en Afrique, elle a cependant pris, ces dernières années, une plus grande ampleur à la suite de conflits déstructurés qui y ont éclos après la guerre froide ${ }^{1}$. «Accueillir des réfugiés [...] est devenu une charge trop lourde à supporter. La protection et l'aide aux réfugiés font encourir de nouveaux risques à la sécurité nationale, exacerbent les tensions entre les États et causent de graves dommages à l'environnement $^{2}$. » Venant d'un responsable tanzanien, un des rares pays africains dont la politique à l'égard des réfugiés a souvent été citée en exemple, cette déclaration montre qu'il y a péril en la demeure et qu'un renouvellement de la réflexion sur la protection des réfugiés et la sécurité régionale en Afrique s'impose.

L'installation des masses importantes de réfugiés aux frontières des pays en guerre, socio-économiquement ou politiquement fragilisés, s'accompagne généralement d'une 
augmentation de la violence, d'une plus grande compétition pour les ressources et de conflits avec les populations locales. Ce sont là autant de ficelles que s'empressent de tirer différents acteurs, tant du pays d'origine que du pays d'asile, pour qui cette masse humaine compactée dans des camps représente un véritable enjeu de pouvoir. Dans le cas de la Guinée, 350 ooo réfugiés, arrivés par vagues successives du Liberia et de la Sierra Leone, sont installés dans 54 camps le long de la frontière avec ces deux pays depuis près de dix ans. La rente humanitaire a permis, pendant des années, d'amortir cette charge. Mais, depuis 1999, la zone d'installation des réfugiés, point de départ ou d'arrivée des incursions armées lancées depuis la Sierra Leone et le Liberia en Guinée et de la Guinée au Liberia, est devenue l'épicentre du tourbillon de la recomposition politique en cours dans cette région. Victimes d'une stratégie qui les dépasse, les réfugiés en paient le prix fort : « traitres » d'un côté de la frontière, « rebelles » de l'autre. La présente note examine les implications normatives et stratégiques de cette situation qui pose un défi majeur au HCR dans l'exercice de sa mission de protection internationale et de recherche de solutions permanentes au problème des réfugiés. Contraire au droit international (III), l'instrumentalisation politique des réfugiés les place au cœur des problèmes vitaux pour la sécurité intérieure (II) et extérieure des États (I).

\section{Réfugiés et sécurité extérieure}

Sur le plan de la sécurité extérieure, les réfugiés représentent un instrument politique aux yeux des mouvements armés qui rêvent de (re)conquérir le pouvoir dans le pays d'origine ${ }^{3}$. Pour ces mouvements, les camps des réfugiés offrent un triple avantage en termes de recrutement, de sanctuaire et de logistique. Soumis aux conditions pénibles de l'exil, les réfugiés entassés dans un camp constituent un "vivier de combattants", recrutés de manière volontaire ou forcée 4 . Il semble ainsi que les dernières attaques du RUF (Revolutionary United Front) sur les camps de réfugiés en Guinée aient visé notamment à procéder à $\mathrm{du}$ « recrutement actif ». Plusieurs réfugiés auraient été pris en otage et ramenés du côté sierra-léonais de la frontières. Par leur situation géographique, leur composition humaine, leur densité démographique et la relative protection dont ils bénéficient en droit international, les camps de réfugiés représentent une base d'appui idéale pour les mouvements armés, une fois leurs objectifs militaires atteints de l'autre côté de la frontière ${ }^{6}$. Il est en effet relativement facile pour les combattants de se dissimuler parmi les populations civiles des camps, surtout lorsque celles-ci parlent la même langue qu'eux ou partagent leur cause politique. En der- nier lieu, l'aide humanitaire déversée dans les camps de réfugiés leur confere une importance logistique considérable. Par différents mécanismes de prélèvement plus ou moins violents, une bonne partie de cette aide peut être recyclée pour soutenir l'effort de guerre? . D'où le dilemme des humanitaires : faut-il continuer à apporter l'aide aux populations civiles en dépit des détournements dont elle est l'objet et qui nourrissent la guerre ${ }^{8}$ ?

La situation des réfugiés rwandais dans l'est de la République démocratique du Congo (RDC) en 1994-1996 se révèle une illustration intéressante de l'instrumentalisation des réfugiés à des fins de reconquête du pouvoir dans le pays d'origine. Après le génocide rwandais de 1994, la RDC a vu déferler sur son territoire près de 1500 ooo réfugiés, sitôt installés dans des camps à l'orée de la frontière entre les deux pays. Dans le cadre d'une stratégie de reconquête rapide du pouvoir perdu à Kigali au profit de la minorité tutsi, les anciennes autorités hutus ont compris, dès le début, l'intérêt qu'il y avait à assurer un contrôle systématique des camps de réfugiés. Primo, elles pouvaient ainsi disposer, à portée de mains, d'un réservoir inépuisable de combattants. Secundo, en raison d'un coût de un dollar par personne et par jour, la masse de réfugiés représentait un gisement dont l'exploitation ne manquait pas d'intérêt. Le détournement d'une partie de l'aide humanitaire dispensée dans les camps servait à soutenir la lutte armée contre le nouveau régime de Kigali. Tertio, les « blindés » arrimés sur la lave volcanique des camps offraient à sa composante militaire un espace de replis aux couleurs de l'humanitaire" Le développement des activités de mouvements armés dans les camps de réfugiés, avec la complicité plus ou moins active des autorités du pays d'asile ne peut qu'entraîner, au mieux, une fermeture des frontières, au pire, des opérations armées transfrontalières conduites, à titre préventif, par le pays d'origine. Il s'agit là d'une constante des relations internationales africaines de ces 30 dernières années ${ }^{10}$. Les nouvelles autorités rwandaises, pour qui la menace des camps représentait un vrai cauchemar, choisiront de les démanteler manu militari en octobre 1996, libérant une onde de choc qui allait traverser toute l'Afrique centrale, et dont les effets se font encore ressentir à ce jour.

\section{Réfugiés et sécurité intérieure}

Sur le plan de la sécurité intérieure, les avantages qu'offrent les camps de réfugiés en termes de recrutement, de logistique et de sanctuaire en font également un enjeu de pouvoir pour les mouvements armés qui combattent les autorités du pays d'asile. Pour rester dans la région des grands lacs africains, la Résistance nationale armée de 
Yoweri Museveni s'était ainsi appuyée, pour une large part, sur les réfugiés tutsi rwandais en Ouganda pour renverser le régime de Kampala. Une fois au pouvoir, Museveni aidera à son tour les anciens réfugiés tutsi, structurés autour du Front patriotique rwandais (FPR), à engager l'assaut final contre le régime de Kigali" ${ }^{11}$ Pour Museveni, cette opération présentait un avantage politique majeur : elle lui permettait de se débarrasser d'un groupe important de réfugiés, désormais aguerris au combat, et sur qui d'autres auraient pu être tentés de s'appuyer un jour. C'est sans doute instruites par leur propre expérience et l'histoire de la reconquête du pouvoir par le FPR que les nouvelles autorités rwandaises ont choisi d'éradiquer, cette fois à l'intérieur des frontières nationales, les camps de personnes déplacées, estimant qu'ils représentaient « un foyer d'hostilité et une menace pour la sécurité intérieure ». C'est au cours de l'une de ces opérations de démantèlement qu'a eu lieu, le 23 avril 1995, le massacre de Kibeho, le plus grand camp de l'ancienne zone humanitaire sûre constituée sous l'opération turquoise, qui avait frustré les nouveaux maîtres de Kigali d'une victoire totale sur les ex-FAR (Forces armées rwandaises) et les Interahamwes (Milices hutus), désormais seigneurs des camps. La même stratégie d'instrumentalisation de réfugiés est présente dans le contexte politique actuel en Guinée, où tout semble indiquer que le mystérieux mouvement d'opposition (Rassemblement des forces démocratiques de Guinée: RFDG), qui a revendiqué les attaques menées aux frontières au mois de décembre dernier, chercherait à prendre avantage des camps de réfugiés qui y sont installés dans son objectif de renversement du régime de Lansana Conté. Dans une remarquable opération de survie politique et de ralliement des masses, ce dernier a, à l'occasion d'un discours prononcé au Palais du peuple le 9 septembre dernier, fustigé « les étrangers qui se sont installés à demeure chez nous et qui nous remercient en apportant la guerre ». Aux yeux de la population guinéenne, une équation simpliste et dangereuse, pouvant ouvrir la voie à tous les abus, court désormais : réfugiés $=$ rebelles.

Il va sans dire qu'une perception aussi négative des réfugiés, qui se généralise en Afrique, ne peut qu'entraîner vers le bas le niveau de protection offerte par les États d'asile. La légendaire « hospitalité africaine » à l'égard des réfugiés tend à s'émousser, et il apparaît de plus en plus évident que l'instrumentalisation politique des réfugiés fait perdre à cette question de son " caractère humanitaire » et risque de remettre en cause tout l'acquis international dans ce domaine. Comme le relève l'ancien haut-commissaire aux réfugiés Sadako Ogata : " Alors que les Africains ont tou- jours été généreux, prompts à accueillir les réfugiés, ils sont en train de changer d'attitude. Aujourd'hui, les arrivants font peur [...] » Il est regrettable qu'après avoir fui son pays d'origine parce que « craignant avec raison » d'être persécuté, pour reprendre les termes de la Convention de Genève, le réfugié inspire à son tour un sentiment de peur à sa communauté d'accueil, plutôt que de la compassion. La peur de l'autre, de la différence, étant à l'origine des comportements les plus extrêmes, il y a lieu de redouter qu'à la crainte de persécution dans le pays d'origine ne puisse alors se substituer la persécution dans le pays d'accueil. Comment enrayer cette évolution dont les conséquences touchent au cœur même du mandat du HCR? Quelles sont les solutions déjà appliquées ou suggérées par le HCR et la communauté internationale dans le domaine de la sécurité? Pour répondre à cette double interrogation, il importe au préalable de dresser l'état du droit international des réfugiés.

\section{III. État du droit international et solutions}

Sur le plan universel, la Convention de Genève relative au statut des réfugiés de 1951 ne consacre pas de disposition particulière à la question de la sécurité. Elle se limite à exprimer « le vœu que tous les États, reconnaissant le caractère social et humanitaire du problème des réfugiés, fassent tout ce qui est en leur pouvoir pour éviter que ce problème ne devienne une cause de tension entre États ${ }^{12}$ ", et à réaffirmer l'obligation générale selon laquelle « tout réfugié a, à l'égard du pays où il se trouve, des devoirs qui comportent notamment l'obligation de se conformer aux lois et règlements ainsi qu'aux mesures prises pour le maintien de l'ordre public ${ }^{13} »$.

C'est sur le plan régional africain que cette question prend un relief particulier. Les problèmes de sécurité liés à l'accueil des grandes masses de réfugiés ont toujours représenté une préoccupation constante des dirigeants africains, même s'il faut constater que les nombreuses résolutions et conférences consacrées à cette question n'ont généralement pas été suivies d'effet. Il suffit, pour s'en convaincre, de considérer la Convention de l'oua régissant les aspects propres aux problèmes des réfugiés en Afrique du 10 septembre 1969, dans laquelle les chefs d'État et de gouvernement se déclarent, dès l'ouverture " conscients de ce que les problèmes des réfugiés constituent une source de friction entre de nombreux États membres, et désireux d'enrayer à la source de telles discordes; désireux d'établir une distinction entre un réfugié qui cherche à se faire une vie normale et paisible, et une personne qui fuit son pays à seule fin d'y fomenter la subversion à partir de l'extérieur; décidés de faire en sorte que les activités de tels éléments 
subversifs soient découragées, conformément à la déclaration sur le problème de la subversion et à la résolution sur le problème des réfugiés, adoptées à Accra en $1965^{14}$ ». Même si la terminologie employée rappelle un âge aujourd'hui révolu, celui de la subversion révolutionnaire, il reste que cette déclaration situe clairement la dimension sécuritaire au cœur de l'instrument régional africain sur les réfugiés qui énonce, par la suite, des obligations fondamentales dans le chef des réfugiés et des États d'accueil, non sans rappeler au préalable le principe selon lequel «l'octroi du droit d'asile aux réfugiés constitue un acte pacifique et humanitaire, et ne peut être considéré par aucun État comme un acte de nature inamicale ${ }^{15} »$. D'une part, tout réfugié doit «s'abstenir de tous agissements subversifs dirigés contre un État membre de l'ous ${ }^{16}$ ». De l'autre, « pour des raisons de sécurité, les États d'asile devront, dans toute la mesure du possible, installer les réfugiés à une distance raisonnable de la frontière de leur pays d'origine ${ }^{17} »$ et " $s^{\prime}$ engagent à interdire aux réfugiés établis sur leur territoire respectif d'attaquer un quelconque État membre de l'ous par toutes activités qui soient de nature à faire naître une tension entre les États membres, notamment par les armes $[\ldots]^{18} »$. Ces dispositions ont-elles été respectées? On peut en douter au vu de l'évolution de la situation des réfugiés en Afrique. Par calculs stratégiques, complaisance ou incapacité des États, les réfugiés sont devenus des otages de jeux politiques peu soucieux de la personne humaine. Qu'il s'agisse de la République démocratique du Congo, du Kenya, de l'Ouganda ou encore de la Guinée, pour ne citer que ces pays, le respect de ces principes eut suffi, à lui seul, à épargner aux États de nombreux problèmes de sécurité attribués, à tort ou à raison, aux réfugiés.

À la faveur de la crise des Grands Lacs en 1992-1996, une vaste réflexion internationale avait été lancée sur la dynamique entre réfugiés et sécurité régionale ${ }^{19}$. Le HCR a pris une part active à cette réflexion et s'est résolument engagée dans la recherche de solutions novatrices au problème de sécurité. Son action aura bénéficié, dans ce domaine, d'un «mandat suffisamment flexible pour permettre le développement d'un véritable espace d'initiative, à l'intérieur duquel l'institution se réinvente constamment ${ }^{20}$ ». Parmi les lignes de force de cette réflexion, qui voudrait donner corps à l'esprit de la Convention de l'oua de 1969 et dans laquelle s'inscrit l'action du HCR, il y a lieu de relever les axes ci-après.

\section{Le désarmement de réfugiés armés}

Les conflits armés étant la cause majeure des réfugiés en Afrique, il arrive souvent que ceux qui sollicitent l'asile fran- chissent la frontière avec armes et munitions. Pour garantir le caractère humanitaire de l'asile, le désarmement de ces groupes demeure une condition primordiale. Cette exigence fait l'objet d'un constant rappel par le HCR, pour qui l'enjeu consiste, sur ce point, à mettre les instances politiques nationales et internationales devant leurs responsabilités en insistant sur le fait que faillir à cette obligation ne peut que déboucher, par la suite, sur des problèmes de sécurité intérieure et extérieure.

\section{La séparation de civils d'avec les combattants}

Le maintien du caractère civil des camps impose de séparer les civils d'avec les combattants. Il s'agit, d'une part, de garantir aux premiers une certaine liberté en empêchant qu'ils ne deviennent les otages des seconds et, d'autre part, d'éviter que les camps ne soient perçus comme une cible militaire $^{21}$. Cette première séparation de type physique devrait être complétée par une autre séparation de type légal, conformément aux clauses d'exclusion des Conventions de Genève et de l'oua, qui écartent du bénéfice de la protection internationale toute personne dont on aurait « des raisons sérieuses de penser » qu'elle a commis un crime contre la paix, un crime de guerre, un crime contre l'humanité, un crime grave de droit commun, ou qu'elle s'est rendue coupable d'agissements contraires aux buts et aux principes des Nations Unies et de l'ouA ${ }^{22}$. Même en contexte d'afflux massif, le recours à la reconnaissance collective du statut de réfugié, qui caractérise la pratique africaine, ne saurait exempter les États d'une application, au moins partielle, des clauses d'exclusion, dont la ratio legis vise à préserver à l'asile sa vraie nature. N'ayant ni mandat ni capacité opérationnelle propre pour entreprendre une séparation physique des réfugiés, le HCR aura centré son action, dans ce domaine, sur deux fronts. D'une part, il s'est investi dans l'apostolat de la parole, en réitérant inlassablement la nécessité d'une telle séparation auprès de ceux qui ont le pouvoir et les moyens de la mettre en ouvre. Même si cette parole n'a pas toujours été entendue, comme dans le cas des réfugiés rwandais à l'est de la République démocratique du Congo, il faut néanmoins reconnaître au HCR le mérite d'avoir su indiquer, à temps et à contretemps, ce qu'il convenait de faire. Le HCR s'est souvent trouvé associé, dans ce rôle, aux responsables de l'oua. De l'autre, il a prêté son concours aux autorités nationales déterminées à entreprendre une telle séparation. Ainsi, par exemple, au titre de ses objectifs principaux en Tanzanie pour l'année 2000, le HCR relève qu'il « continuera d'assister les autorités tanzaniennes par une série de mesures. Il soutiendra notamment le déploiement et la formation de contingents

(C) Jack M. Mangala, 2001. Cette œuvre en libre accès fait l'objet d'une licence Creative Commons Attribution-NonCommercial 4.0 International License, laquelle autorise l'utilisation, la reproduction et la distribution de l'œuvre sur tout support à des fins non commerciales, pourvu que

l'auteur ou les auteurs originaux soient mentionnés et que la publication originale dans Refuge : revue canadienne sur les réfugiés soit citée. 
de polices spéciaux et la mise en place d'installation permettant de séparer les combattants de la population réfugiée, afin de préserver le caractère civil et humanitaire des camps de réfugiés ${ }^{23} »$.

\section{L'installation des camps loin de la frontière avec le pays d'origine}

La localisation des camps loin de la frontière avec le pays d'origine offre un double avantage en termes de sécurité. Elle les rend moins vulnérables aux attaques d'éléments venus du pays d'origine et permet, par la zone tampon ainsi créée, de mieux contrôler les flux d'activités transfrontières en direction et au départ des camps. À ce propos, le Comité exécutif du programme du HCR rappelle que «le lieu de séjour des personnes en quête d'asile doit être déterminé en fonction de leur sécurité et de leur bien-être ainsi que des exigences de sécurité de l'État d'accueil ${ }^{24}$ ». Pour des raisons géopolitiques ou socio-économiques, la mise en œuvre de ce prescrit n'a pas toujours rencontré l'assentiment des autorités nationales. Le HCR a néanmoins entrepris, dans les pays où la coopération des autorités nationales lui était acquise, des programmes de transfert des réfugiés plus à l'intérieur du pays. Près de 15000 réfugiés sierra-léonais de Guinée ont ainsi été transférés en 1999, et il était prévu d'en faire de même pour 65000 autres ${ }^{25}$. Certains chercheurs commencent toutefois à se demander si l'on ne gagnerait pas, tant sur le plan de la sécurité que sur le plan humanitaire, à laisser les réfugiés s'installer librement parmi les populations locales, plutôt qu'à les confiner dans des camps $^{26}$. Lorsque cela est possible, le libre choix du lieu de résidence demeure un mode d'installation préférable. Quand les circonstances ne s'y prêtent pas, il faudrait néanmoins veiller à ce que les camps gardent une dimension humaine, susceptible d'en faciliter l'administration autant que la sécurisation.

\section{La sécurisation des camps}

La question de la sécurisation des camps comporte une double dimension. Il s'agit, d'une part, d'éviter que les camps ne soient l'objet d'attaques armées et, d'autre part, de veiller à ce qu'ils ne se transforment en zones de nondroit, livrées à la violence et aux intimidations. Pendant longtemps, la communauté internationale ne s'est préoccupée que de la dimension externe de la sécurité des camps, à laquelle l'Exсом a consacré de nombreuses conclusions qui, sur un mode répétitif, se limitent pour l'essentiel à condamner les attaques armées contre les camps ${ }^{27}$. La proposition formulée par l'ancien haut-commissaire aux réfugiés, Poul Hartling, de voir les gouvernements s'accor- der pour inclure expressément les camps de réfugiés parmi les catégories juridiquement protégées par les Conventions sur le droit de la guerre n'aura suscité que peu d'intérêt ${ }^{28}$. C'est la crise des réfugiés dans la région des Grands Lacs qui a permis de porter l'attention sur la dimension interne du problème. Il convient à ce sujet de rappeler que la sécurité dans et aux alentours des camps repose d'abord sur la responsabilité de l'État d'asile, que c'est à lui qu'il revient de faire éventuellement appel à la communauté internationale pour l'aider à assumer cette tâche. Dans son Rapport sur les causes des conflits et la promotion d'une paix et d'un développement durable en Afrique, le secrétaire général de l'ona consacre une importante section au problème de la sécurisation des camps et des aires de refuge. Après avoir rappelé que « la protection des réfugiés et l'aide aux États d'asile requièrent parfois un pouvoir d'intervention que ne possèdent pas les dispensateurs de secours, touchant souvent à des questions de paix et de sécurité internationales qui sont essentiellement du ressort du Conseil de sécurité », le secrétaire général « souhaite vivement que l'on crée un dispositif international qui permette d'aider les pays d'asile à maintenir la sécurité et la neutralité dans les camps ou les zones de regroupement des réfugiés ${ }^{29} »$. Bien qu'aucune piste n'ait été avancée par le responsable onusien quant à la faisabilité de sa proposition, l'idée mérite approfondissement.

C'est dans le domaine de la sécurisation des camps que le HCR a développé, ces dernières années, une gamme d'initiatives nouvelles et intéressantes, en collaboration avec les pays d'asile et ses partenaires opérationnels. Ainsi, pour maintenir la sécurité dans les camps du Zaïre (République démocratique du Congo), le HCR et ce pays ont adopté, le 27 janvier 1995, un «aide-mémoire » prévoyant le recours à un contingent zaïrois encadré par des experts internationaux ${ }^{30}$. Aux termes de cet accord, le Contingent zaïrois pour la sécurité (CZsc) avait, entre autres missions, à assurer le respect de la loi et de l'ordre public dans et autour des camps, et de prévenir les intimidations et la violence contre les candidats au retour au Rwanda ${ }^{31}$. Des accords similaires de sécurisation de camps ont également été conclus avec la Tanzanie et le Kenya ${ }^{32}$. Dans ce dernier pays, les efforts du HCR visaient à résorber l'insécurité dans les zones d'installation de réfugiés de Kakuma et de Dadaab au nordouest. Parmi les dispositions prises à cette fin, l'on retiendra principalement : le soutien aux forces de police locale, par le renforcement de leur capacité et de leur efficacité; l'introduction des arrangements de sécurité dans les camps, à travers notamment le recrutement des anciens officiers de l'armée kenyane comme coordonnateurs de sécurité, et 
la création d'une force de 120 gardes locaux comprenant réfugiés et populations locales; la réorganisation de la vie communautaire dans les camps afin d'impliquer davantage les réfugiés aux questions de sécurité; certaines actions particulières visant les groupes vulnérables tels que les femmes réfugiées ${ }^{33}$.

\section{La coopération régionale}

Les implications des problèmes de réfugiés sur le plan de la sécurité plaident en faveur d'une approche régionale renouvelée dans la recherche de solutions. Comme l'a reconnu la Conférence régionale sur les questions de réfugiés dans la région des Grands Lacs, "the countries of the Great Lakes have common interests and common destiny". Ce qui est vrai pour cette région du continent africain, l'est également pour d'autres. Quand survient un problème de réfugiés, il est important que tous les pays intéressés, au premier rang l'État d'origine et l'État d'asile, établissent des mécanismes réguliers de consultation et de coopération, et s'accordent sur un ensemble de mesures destinées à inspirer confiance à toutes les parties. Parmi ces mesures, l'institution de commissions de sécurité impliquant également les représentants des réfugiés, le HCR et d'autres agences humanitaires pourrait se révéler un rouage institutionnel particulièrement utile.

\section{L'assistance internationale}

L'application des prescrits minimaux énumérés dépasse souvent les capacités des États d'asile africains, déjà englués dans des problèmes socio-économiques et politiques inextricables. De ce fait, l'impératif de sécurité représente une invitation à une solidarité internationale plus agissante dans l'esprit du préambule de la Convention de Genève de 1951 relative au statut des réfugiés, qui énonce "qu'il peut résulter de l'octroi du droit d'asile des charges exceptionnellement lourdes pour certains pays et que la solution satisfaisante des problèmes dont l'organisation des Nations Unies a reconnu la portée et le caractère internationaux ne saurait, dans cette hypothèse, être obtenue sans une solidarité internationale ». C'est à la même solidarité internationale que le HCR et l'ouA en ont également appelé dans le document d'Addis Abeba sur les réfugiés et les déplacements forcés de population en Afrique ${ }^{34}$.

\section{Considérations finales}

Le droit international des réfugiés repose sur une double préoccupation : protéger les droits des réfugiés et garantir les intérêts légitimes des États. Parmi ces intérêts, la Convention de Genève, dans une moindre mesure, et la Con- vention de l'ous, de manière plus soutenue, mettent en avant la sécurité. Ces dernières années, l'instrumentalisation politique des réfugiés a accru les problèmes de sécurité aussi bien à l'intérieur des États d'asile que dans les relations interétatiques. Cette situation, qui risque de remettre en cause tout l'acquis international dans le domaine de la protection des réfugiés, représente un important défi pour le HCR. Ce dernier essaie, dans les limites de son mandat et les contraintes pesant sur ses capacités opérationnelles, d'y répondre par une série d'initiatives qui donnent la mesure d'une remarquable capacité d'innovation. Au-delà de l'action ponctuelle du HCR, dont l'impact ne saurait être en la matière que limité, il importe que les acteurs engagés dans des luttes de pouvoir redécouvrent les vertus de l'esprit humanitaire de l'asile et s'engagent enfin au respect du droit. Quod non, la montée des préoccupations sécuritaires risque de sonner le glas de l'institution de l'asile et de la protection des réfugiés en Afrique ${ }^{35}$.

\section{Notes}

1. Les conflits déstructurés se caractérisent par l'atomisation de l'autorité et la méconnaissance des normes élémentaires de droit humanitaire de la part de belligérants. Ils entraînent de ce fait un grand nombre de réfugiés et de personnes déplacées.

2. E. Mwanbulukutu, vice-ministre tanzanien de l'intérieur, cité dans Augistine Mahiga, "Changement de cap en Tanzanie » (1997), hiver Réfugiés, 15.

3. Voir : Christophe Comblin, Les réfugiés, instrument politique dans le tiers-monde (Bruxelles : GRIP, 1995).

4. Au temps de l'apartheid, les réfugiés namibiens d'Angola représentaient un réservoir important de combattants pour la sWAPO. De même, l'AnC recrutait massivement dans les camps du Botswana, du Mozambique et du Zimbabwe. Voir : Philippe Chapleau, «Les réfugiés dans les conflits d'Afrique australe » (1987), 44 Études polémologiques, 93. Le statut particulier de ces mouvements de libération, qui bénéficiaient du soutien des membres de l'oua, mérite d'être relevé. Dans la corne de l'Afrique, les différents mouvements armés ont eu recours, pendant des années, aux camps de réfugiés pour renouveler leurs troupes. Voir : René Otayek, « Du bon usage du réfugié dans la corne de l'Afrique : quelques remarques » (1987), 44 Études polémologiques, 77-86.

5. Le Monde, 26 décembre 2000.

6. Voir : Jean-Christophe Rufin, Le piège humanitaire (Paris : Hachette, coll. Pluriel, 1993), 125-128.

7. À titre indicatif, à l'occasion de la reprise des combats au Liberia en avril 1996, les équipes humanitaires présentes dans ce pays ont perdu en quelques semaines pour plus de 20 millions de dollars d'équipement, dont près de 500 véhicules. Voir : Fabrice Weissman, "Le Liberia, otage des seigneurs de la guerre », dans Médecins Sans Frontières, Populations en danger (Paris : La Découverte, 1996), 95-110.

8. En 1994 par exemple, le retrait de Médecins Sans Frontières des 
camps de réfugiés du Kivu fut motivé par le refus de participer, indirectement, à la reconstruction des forces responsables du génocide. Cette décision ne fut pas partagée par les autres ONG qui continuèrent leurs activités humanitaires dans les camps.

9. Dans les camps du Kivu, le terme "blindé » désignait l'habitat de toile plastique bleue, blanche ou verte des réfugiés.

10. Du temps de l'apartheid, les pays de la ligne de front ont ainsi payé un lourd tribut à leur soutien aux mouvements de libération. Les camps de réfugiés ont constamment été l'objet d'attaques des forces spéciales sud-africaines.

11. Voir : Roland Pourtier, "La guerre au Kivu : un conflit multidimensionnel » (1996), 4 Afrique Contemporaine, 33 et 37.

12. Préambule, troisième considérant.

13. Article 2.

14. S. 3,4 et 5 du préambule.

15. Article II, s. 2.

16. Article III, s. 1.

17. Article 11, s. 6. Qu'entend-on par " distance raisonnable »? Il y a très peu d'indications à ce sujet. Le directeur des opérations du HCR pour l'Afrique occidentale, orientale et centrale l'évalue à $50 \mathrm{~km}$. Voir Interview dans Jeune Afrique, 4 février 1997, 24. Cette mesure d'installation est recommandée " for obvious security reasons, as much for the sake of the refugees themselves as for countries of origin and of refuge », conférence Legal, Economic and Social Aspects of African Refugee Problems (9-18 octobre 1967), rapport final, doc. AFR/REF/CONF.1967, p. 26.

18. Article III, s. 2. Il sied, à cet égard, de rappeler le statut particulier dont bénéficiaient les mouvements de libération combattant les régimes minoritaires et racistes, non-membres de l'ouA. Dans sa Déclaration sur le problème de la subversion, doc. AHG/ Res. 27 (II), 25 octobre 1965, la Conférence des chefs d'État et de gouvernement s'était engagée " à continuer à garantir la sécurité des réfugiés politiques (des territoires africains non indépendants) et à leur accorder soutien dans la lutte pour la libération de leur pays $"$.

19. Voir notamment : « Refugee protection and security in the great lakes region », rapport du Regional Meeting on Refugee Issues in the Great Lakes (Kampala, Ouganda, 8 et 9 mai 1998), Refugee Survey Quarterly, vol. 17, ${ }^{\circ}$ 2, 1998, 1-45.

20. Jean-Francois Durieux, «Le rôle du Haut-commissariat des Nations Unies pour les réfugiés ", Société française pour le droit international (Éd.), Droit d'asile et des réfugiés, colloque de Caen (Paris : Pedone, 1997), 192.

21. Voir: HCR, The Impact of Military Personnel and the Militia Presence in Rwandese Refugee Camps and Settlements, doc. 1995/ Buj, conf. 3, 1995. «In situations where the national authorities lack the capacity to take such action [separating refugees from other exiles], alternative approaches might be considered including the deployment of regional or international police and military forces ", Refugee Protection and Security in the Great Lakes Region, rapport du Regional Meeting on Refugee Issues in the Great Lakes (Kampala, Ouganda, 8 et 9 mai 1998), op. cit., 6. Dans le cas de la République démocratique du Congo, il y a eu une proposition de l'ancien ministre belge des affaires étrangères, Leo Tindemans, de casernement des ex-FAR au camp militaire de Kamina dans la région du Shaba. Ce projet n'a pas obtenu l'adhésion des autorités congolaises qui n'ont pas voulu assumer les risques liés à la présence d'une importante force combattante à l'intérieur du territoire.

22. Articles $1 \mathrm{~F}$ de la Convention de Genève et $1 \mathrm{~s} .5$ de la Convention de l'ous. En droit international des réfugiés, l'application des clauses d'exclusion repose sur une simple présomption. Il suffit que les autorités du pays d'asile aient « des raisons sérieuses de penser que [...] » Voir le supplément que l'International Journal of Refugee Law, vol. 12, hiver 2000, consacré aux clauses d'exclusion. Pour un examen de la pratique africaine, voir spécialement William O'Neill, Bonaventure Rutinwa and Guglielmo Verdirame, «The Great Lakes : A Survey of the Application of the Exclusion Clauses in the Central Africa Republic, Kenya and Tanzania », 135-170; William O'Neill, " Conflict in West Africa : Dealing with Exclusion and Separation », 171-194. 23. HCR, Appel global 2000 (Genève : HCR, 1999), 55.

24. Conclusion $\mathrm{n}^{\circ} 22$ (xxxII).

25. Ibid., 93. Quoique significatives, ces opérations de déplacement sont cependant restées insuffisantes.

26. Voir : Jeff Crisp and Karen Jacobsen, «Refugees camps reconsidered" (1998), 3 Forced Migration Review, 27-30; Collette Braeckman, «Les camps? Pas toujours la meilleure solution ", Le soir, 21 août 1995.

27. Voir les Conclusions $n^{\circ} 27$ (xxxiII), $n^{\circ} 32$ (xxxiv), $n^{\circ} 45$ (xxxviI) et $\mathrm{n}^{\circ} 48$ (xxxviII) ainsi que la Note sur les attaques armées contre les camps et zones d'installation de réfugiés, doc. $\mathrm{EC} / \mathrm{sCP} / 47,10$ août 1987.

28. Même si les camps ne bénéficient pas, en tant que tels, d'une consécration expresse comme catégorie juridiquement protégée, il y a lieu de relever cependant que la présence de combattants au sein de groupes de réfugiés strictement civils ne prive pas ces derniers de toute protection. D'une part, les attaques indiscriminées qui frapperaient aussi et a fortiori surtout les civils restent interdites par les articles 51 et 57 du Protocole I aux Conventions de Genève. D'autre part, en vertu de l'article 50 s. 1 du même Protocole, la présence au sein d'une population civile, et par extension d'une population de réfugiés, de combattants isolés ne prive pas cette population de sa qualité.

29. Doc. Nus/1998/318, 16 avril 1998, s. 55. L’ancien secrétaire général de l'onu Boutros Ghali avait, dans son deuxième Rapport sur la sécurité des camps de réfugiés rwandais, doc. S/1995/65, 25 janvier 1965, proposé la mise sur pied d'une opération de maintien de la paix ou, à défaut de celle-ci, l'adoption de l'une des trois solutions de rechange suivantes: la mise sur pied d'un groupe de policiers/observateurs internationaux, la conclusion d'arrangements contractuels avec des organismes privés de sécurité ou l'adoption des mesures de sécurité par l'intermédiaire du HCR. C'est la dernière option qui fut finalement retenue.

30. Texte disponible au Centre de documentation et de recherche du HCR, Genève.

31. Voir : Mutoy Mubiala, «Les Nations Unies et la crise des réfugiés rwandais » (1996), 2 Revue belge de droit international, 493515.

32. Textes disponibles au Centre de documentation et de recherche du HCR, Genève.

33. Voir : Jeff Crisp, «A state of insecurity : The political economy 
of violence in refugee-populated areas in Kenya » (1999), en ligne : <http://www.unhcr.ch> (date d'accès : 28 février 2001).

34. Voir la Recommandation $n^{\circ} 8$, qui relève notamment que l'assistance internationale devrait « permettre aux gouvernements de faire face efficacement aux situations pouvant contribuer à une dégradation de la sécurité, du droit et de l'ordre public dans les zones accueillant les réfugiés".

35. Voir Bonaventure Rutinwa, "The end of the asylum? The changing nature of refugee policies in Africa » (1999), en ligne : $<$ http://www.unhcr.ch> (date d'accès : 28 février 2001).

Docteur en droit de l'Université catholique de Louvain (Belgique), Jack M. Mangala enseigne actuellement à Grand Valley State University, MI, USA. L'auteur termine une recherche financée par l'OTAN sur le déplacement forcé de population comme nouvelle dimension de la sécurité. Il compte plusieurs articles dans les journaux spécialisés en droit des réfugiés.

(C) Jack M. Mangala, 2001. Cette œuvre en libre accès fait l'objet d'une licence Creative Commons Attribution-NonCommercial 4.0 International License, laquelle autorise l'utilisation, la reproduction et la distribution de l'œuvre sur tout support à des fins non commerciales, pourvu que l'auteur ou les auteurs originaux soient mentionnés et que la publication originale dans Refuge : revue canadienne sur les réfugiés soit citée. 